\title{
EDITORIAL
}

\section{Design of Multidisciplinary Cyber Physical Systems}

\author{
Sofiane Achiche $a^{a^{*}}$ and Tetsuo Tomiyama ${ }^{b}$ \\ ${ }^{a}$ École Polytechnique de Montéral, Québec, Canada \\ ${ }^{b}$ Cranfield University, Bedfordshire, UK
}

A cyber-physical system (CPS) is a system of collaborating computational elements that control and interact with physical entities. These systems can be found in areas such as aerospace, automotive, energy, healthcare, manufacturing, entertainment, and consumer appliances, and can also be categorized as embedded systems, mechatronics, and smart X. In embedded systems the emphasis is on the computational elements, while mechatronic systems focus on the link between the computational and physical elements in terms of mechanical, electronics, control engineering and computer science domains. The design of cyber physical systems (mechatronics, embedded systems, smart $\mathrm{X}$, etc.) is quite distinctive from any other designs, because they address highly complex multidisciplinary issues related to the combination of product/system/service (Rauniyar \& Tanik, 2010). The inherent complexity in the design task due to the multidisciplinary aspects of CPS put high stress on engineers' creativity to produce more innovative solutions. These challenges are known by the Engineering Design community in mechatronics (TorrySmith et al., 2013) but are exacerbated by the added layers of the cyber communication between the different objects, as well as the several challenges of possible IT service design which are of a different engineering nature (Dargahi et al., 2012; Wang, 2012). Therefore, assisting design engineers in design tasks as well as reducing the intrinsic complexity of the modeling is of crucial importance. This can be achieved by either design support tools or new methodologies that would make the design easier and simpler. The papers in this this special issue present the state of the art and advanced techniques related to the development, modelling and simulation of complex CPS. We cover controller design using system architecture, video servoing of robotic systems as well as characterisation of an instrumented dynamic flexible structure.

The first paper written by Alvarez Cabrera and Tomiyama entitled "Architecture-Level Representation and Analysis of Regulatory Controller Configuration for Complex Mechatronic Systems" provides a method for automatically extracting information that is relevant for the controller design activities from a model that represents architecture-level information and concerns, and shows how the extracted information can be analyzed for control design purposes. Control design is closely linked to several subsystems within the cyber physical system, and the authors put architecture-level models at the core of the design activities of the control systems. The main contributions of this paper are the use of an architecture model and the extraction of information relevant to control design from it, while the control analysis process is taken from existing linear structured system techniques. The authors' work provides great insight into how to improve control design, an inherently multidisciplinary, from improvements in the communication and exchange of updated design information between stakeholders during product development of CPS.

The second paper, entitled "A Comparative Study on Eye-In-Hand Image-Based Visual Servoing; Stereo vs. Mono" written by Mohebbi et al. presents an application in visual servoing robotics, where

* Corresponding author. Email: sofiane.achiche@polymtl.ca. 
several mechatronics systems collaborate for a given task to form a CPS. More specifically a comparative study of eye-in-hand image-based visual servoing (IBVS) for two approaches of stereo and mono vision systems is presented and various cases of scenarios, control schemes, and tracking and prediction algorithms are studied. In this study the vision systems are mounted on the end-effector of a 6 DOF manipulator robot. Additionally, a method for position prediction and trajectory estimation of the moving target in order to use in a real-time catching task is proposed and developed using Kalman Filter and Extended Kalman Filter as the trajectory estimators. This work proposes the application of a novel Acceleration Command-based controller to the visual servoing systems. The results for both cases are presented to compare the effects on the quality of the servoing tasks.

The third paper, entitled "An Architecture-Level Specification for Automated Supervisory Controller Design" written by Alvarez Cabrera et al., proposes a supervisory control design process that merges descriptions at the level of the system architecture with specific design methods related to supervisory control synthesis. The goal of supervisory control is to ensure a desired process behavior within constraints (e.g., safety or cost). In contrast to the supervisory task, the authors define the regulatory task as directly ensuring that a controlled variable or parameter remains close to a desired reference value, in the context of a single set of continuous system dynamics. The advantages of this Automated Supervisory Controller proposal are a better integration of the supervisory control design with other design processes and stakeholders, improving the potential to automate part of the design process, and a complete specification in a modeling language that remains independent of the particular language of code implementation. The authors conclude that aside from the interest for modeling, the proposed approach is more effective, is more objective, than what exists and can be used for engineering project effectiveness evaluation activities.

The fourth paper, entitled "Adaptive Neuro-Fuzzy Inference System Models for Force Prediction of a Mechatronic Flexible Structure" written by Achiche et al., tackles the cyber physical systems design on the application side. The research describes the results obtained from a research work investigating the performance of different Adaptive Neuro-Fuzzy Inference System (ANFIS) models developed to predict excitation forces on a dynamically loaded flexible structure. Two types of models were developed; the first type uses current accelerations only while the second type considers both the current accelerations and the historical values. The frequency domain is considered to analyse the similarity of the frequencies in the predicted and the original force signal. For a selection of the best models, a more advanced performance analysis is carried out. This includes application of the trained models to deterministic and nondeterministic excitation forces with different excitation frequencies and amplitudes. The results obtained in this paper show that ANFIS models can be used to set up reliable force predictors for dynamical loaded flexible structures, when a certain degree of inaccuracy is accepted. Furthermore, the comparison study points out that the transducer location is crucial for the model performance. However, there exists no general solution for the final selection of models. The findings showed that the model type employing historical values gives better predictions when operating in their trained regions, while the models using only current values have generally higher prediction errors in trained regions but are less sensitive to changes of the system dynamics history.

\section{References}

Dargahi, F., Wang, C., Bhuiyan, M. F. H., \& Mehrizi, H. (2012). Agent-Based System Design for Service Process Scheduling: Challenges, Approaches and Opportunities. Journal of Integrated Design \& Process Science, 16(2), 15-32.

Rauniyar, D.\& Tanik, U. J. (2010). Integrated Product Development Approach for Cyber-Physical Systems Utilizing Standardized Modeling Languages and Methodologies. Journal of Integrated Design \& Process Science, 14(3), 1-11.

Torry-Smith, J. M., Qamar, A., Achiche, S., Wikander, J., Mortensen, N. H., \& During, C. (2013). Challenges in Designing Mechatronic Systems. Journal of Mechanical Design, 135(1), 011005. 
Wang, C. (2012). Editorial: Tackling the Challenges of IT Services Design. Journal of Integrated Design \& Process Science, 16(2), 1-3. doi: 10.3233/jid-2012-0014

\section{Author Biographies}

Dr. Sofiane Achiche has been a professor at École Polytechnique de Montréal, Department of Mechanical Engineering since December 2011, he received the M.Sc.A. and Ph.D. degrees from Polytechnique Montréal, Canada. His research interests focus upon evolutionary computational intelligence applied to engineering problems such as condition monitoring. Furthermore, he works in the field of mechatronics design as well as understanding and modeling activities of new product development processes for decision support purposes. Prior to his appointment at Polytechnique Montréal, Dr. Achiche worked as an associate professor at the Technical University of Denmark in Engineering Design from 2006 to 2011. He was part of the Product Development Group at the Mechanical Engineering Department.

Dr. Tetsuo Tomiyama has been Professor of Life Cycle Engineering at the Manufacturing Department of Cranfield University in the UK since October 2012. Prior to this appointment, he was a professor at Delft University of Technology in the Netherlands between 2002 and 2012, a professor and an associate professor at the University of Tokyo in Japan between 1996 and 2000 and between 1987 and 1996, respectively. Professor Tomiyama is an internationally well-known expert in design theory and methodology, function modelling, complex systems architecting, maintenance engineering, and service engineering. He has pioneered in such topics as intelligent CAD, self-maintenance machines, life cycle simulation, and service CAD. He has been awarded a number of prestigious awards including Royal Society Wolfson Research Merit Award in 2012. He is a fellow of SDPS, ASME, CIRP (The International Academy for Production Engineering) and JSME (The Japan Society of Mechanical Engineers). He holds BEng, MEng, and PhD all from the University of Tokyo. 